\title{
Management Dilemmas in the Treatment and Follow-Up of Advanced Juvenile Nasopharyngeal Angiofibroma
}

\author{
Andrew H. Marshall Patrick J. Bradley \\ Department of Otolaryngology, Head and Neck Surgery, Queen's Medical Centre NHS Trust, \\ University Hospital, Nottingham, UK
}

\section{Key Words}

Juvenile nasopharyngeal angiofibroma $\cdot$ Midfacial

degloving $\cdot$ Pre-operative embolization

\begin{abstract}
Advanced juvenile nasopharyngeal angiofibroma (JNA) (i.e. disease with intracranial extension) is reportedly uncommon. The optimum management form of such a clinical situation currently remains controversial. This review, of the recent English published literature, discusses the common JNA classification systems advocated, shows the advantages and disadvantages of surgical approaches used (both open, endoscopic or combined), and re-evaluates the current role of radiotherapy (for initial management or treatment of residual/recurrent disease). We also discuss the typical presentation of these patients, the role of pre-operative embolization, as well as surgical techniques to minimize recurrence, and advocate a post-treatment follow-up protocol.
\end{abstract}

Copyright $(\subset 2006$ S. Karger AG, Basel

\section{Introduction}

Juvenile nasopharyngeal angiofibroma (JNA) is a rare, benign tumour of predominantly adolescent males. Debate persists about the optimum way to manage advanced disease (i.e. disease with intra-cranial extension). The traditional options of open surgery with/without radiotherapy have recently been challenged by use of the endoscopic approach. A literature review was conducted (in the last 6 years) in an attempt to clarify the current opinions and recommendations - the advantages and disadvantages of the various options available.

\section{Classifications for JNA}

Three classification systems are in current common use, including Fisch's [1], Chandler's [2], and Radowski's [3] adaptation of Sessions' classification [4]. In order to interpret the results discussed below, we included a description of each of these systems (tables 1-3).

Most authors accept the origin of JNA tumour to be the superior aspect of the sphenopalatine foramen [5]. Lloyd et al. [6] analysed the radiological appearance of 72 cases of JNA and believed the origin to be in the pterygo-

\section{KARGER}

Fax +4161306 1234 E-Mail karger@karger.ch www.karger.com
Mr. Patrick J. Bradley MBA, FRCS

Department of Otolaryngology, Head and Neck Surgery

Queen's Medical Centre NHS Trust, University Hospital

Nottingham, NG7 2UH (UK)

Tel. +44 115924 9924, ext. 35514, Fax +44 115970 9748, E-Mail pjbradley@zoo.co.uk 
Table 1. Chandler's classification

\begin{tabular}{ll}
\hline Stage & Tumour extent \\
\hline I & Confined to the nasopharynx \\
\hline II & Extends to the nasal cavity and/or sphenoid \\
\hline III & $\begin{array}{l}\text { Extends to one or several of the following: antrum, } \\
\text { ethmoids, pterygomaxillary and infratemporal fossa, } \\
\text { orbit and/or cheek }\end{array}$ \\
\hline IV & Extends into the cranial cavity \\
\hline
\end{tabular}

Table 2. Andrew's (modified Fisch) classification

\begin{tabular}{ll}
\hline Type & Tumour extent \\
\hline I & $\begin{array}{l}\text { Limited to the nasopharynx and nasal cavity, bone } \\
\text { destruction negligible, or limited to the sphenopalatine } \\
\text { foramen }\end{array}$ \\
\hline II & $\begin{array}{l}\text { Invades the pterygopalatine fossa or the maxillary, } \\
\text { ethmoid or sphenoid sinus with bone destruction }\end{array}$ \\
\hline IIIa & $\begin{array}{l}\text { Invades the infratemporal fossa or the orbital region } \\
\text { without intracranial involvement }\end{array}$ \\
\hline IIIb & $\begin{array}{l}\text { Invades the infratemporal fossa or orbit with } \\
\text { intracranial extradural (parasellar) involvement }\end{array}$ \\
\hline IVa & $\begin{array}{l}\text { Intracranial intradural tumour without infiltration of } \\
\text { the cavernous sinus, pituitary fossa, or optic chiasm }\end{array}$ \\
\hline IVb & $\begin{array}{l}\text { Intracranial intradural tumour with infiltration of the } \\
\text { cavernous sinus, pituitary fossa, or optic chiasm }\end{array}$ \\
\hline
\end{tabular}

Table 3. Radowski's modification of Sessions' classification

\begin{tabular}{ll}
\hline Stage & Tumour extent \\
\hline Ia & $\begin{array}{l}\text { Limited to the posterior nares and/or nasopharyngeal } \\
\text { vault }\end{array}$ \\
\hline Ib & $\begin{array}{l}\text { Involving the posterior nares and/or nasopharyngeal } \\
\text { vault with involvement of at least one paranasal sinus }\end{array}$ \\
\hline IIa & Minimal lateral extension to the pterygomaxillary fossa \\
\hline IIb & $\begin{array}{l}\text { Full occupation of the pterygomaxillary fossa with or } \\
\text { without superior erosion orbital bones }\end{array}$ \\
\hline IIc & $\begin{array}{l}\text { Extension into the infratemporal fossa or extension } \\
\text { posterior to the pterygoid plates }\end{array}$ \\
\hline IIIa & $\begin{array}{l}\text { Erosion of the base of the skull (middle cranial fossa/ } \\
\text { base of pterygoids) - minimal intracranial extension }\end{array}$ \\
\hline IIIb & $\begin{array}{l}\text { Extensive intracranial extension with or without } \\
\text { extension into the cavernous sinus }\end{array}$ \\
\hline
\end{tabular}

palatine fossa, in the recess behind the sphenopalatine ganglion at the exit of the pterygoid canal. These tumours are most likely androgen dependent, explaining their almost exclusive presentation in males, and they may arise from a residual vascular plexus from the involution of the first branchial arch artery [7]. This would explain the fact that although the majority of tumours derive their blood supply from the external carotid system, it is possible for tumours to derive their supply from the internal carotid system.

\section{Presenting Symptoms}

The most frequently reported presenting symptoms in all the series reviewed were nasal obstruction with/without epistaxis. Other associated symptoms include cheek swelling, proptosis, anosmia, weight loss, nasal discharge, impaired hearing, and change in voice [3, 8]. These symptoms were often present for up to 12 months before the diagnosis of JNA was made $[3,9,10]$.

\section{Treatment Options}

It is agreed that surgical excision is the treatment of choice for JNA. The surgical approach should be selected according to the location and the extent of the tumour, the tumour vasculature, the effectiveness of the embolization, the age of the patient, and the experience of the surgical team [11]. The use of adjuvant treatments must be based on clinical experience, a full discussion with the treating clinicians, and thereafter, with complete patient and parental informed consent as to the intended consequences.

\section{Open Surgery}

Traditional surgical approaches include transpalatal, lateral rhinotomy and the use of midfacial degloving. The transpalatal approach has fallen out of favour as it affords poor exposure for large tumours, resulting in a greater chance of recurrence $[3,8,9]$. The use of the midfacial degloving approach has become increasingly popular, because it avoids any facial scars, in comparison with lateral rhinotomy $[12,13]$. Andrews et al. [1] advocate the infratemporal approach for large tumours, whilst others propose a combined anterior and lateral approach [10, 11]. 
Daniesi et al. [12] reported their experience using either a lateral rhinotomy or midfacial degloving approach for JNA that had an associated intracranial extension (Fisch IIIb-IV). They were able to achieve complete tumour resection in 11 of 14 cases (78\%). At the time of surgery, the tumours were located extra-durally and were medial to the cavernous sinus, which resulted in the supero-lateral displacement of the internal carotid artery. These findings permitted a surgical plane of dissection to be followed, which looked unlikely on the pre-operative radiology. In 3 cases, complete tumour excision had not been achieved, 2 cases had complete excision at a second procedure, and the third patient had no further treatment and demonstrated no evidence of growth on imaging at follow-up 6 years later.

Zhang et al. [14] reported Fisch's recent experience using the infratemporal fossa approach and stated that the type $\mathrm{C}$ approach is necessary to treat advanced tumours (Fisch IIIb and IV). They reported on 16 patients who underwent such an approach, as well as 4 patients with smaller tumours who underwent the type D approach. In 4 cases, the tumour was deliberately not removed, as it was located within the cavernous sinus; it was felt at the time by the surgeons that the likely resultant morbidity (blindness) from complete removal would be unacceptable. These residual tumour cases demonstrated no growth or change in size over a follow-up period ranging from 46 to 53 months. The infratemporal fossa approach results in a maximal conductive hearing loss on the operated side. The mandibular and possibly maxillary divisions of the trigeminal nerve are sacrificed using this approach; however, none of the patients reported permanent facial anaesthesia, although it was remarked that nerve recovery might not have achieved the pre-operative level. The resultant surgical bone defect is replaced by the transposed filled temporalis muscle flap, which results in a temporal cosmetic depression defect.

Bales et al. [10] have employed a combined infratemporal fossa and facial translocation approach to the treatment of extensive tumours (Radowski's IIIb). They describe their experience of 5 cases, stating that the cavernous carotid artery required dissection in 3 cases. The morbidities associated with the approach were nasal crusting, facial anaesthesia (2 cases), transient facial lymphoedema, intermittent eye irritation, and malodorous nasal discharge (1 case). It was felt that the morbidities were acceptable in dealing with such advanced disease. One case had a recurrence 19 months after the resection, which was treated by radiotherapy.

Treatment and Follow-Up of Advanced JNA

\section{Combined Open and Endoscopic Surgery}

Several authors have combined the open approach with endoscopic techniques to allow close inspection of the surgical cavity and to allow for further resection, if necessary. Herman et al. [15] expressed the opinion that the use of the endoscope in this combined fashion decreases the likelihood of tumour recurrence. El-Banhawy et al. [16] performed endoscopically assisted excision via a midfacial degloving approach in 15 cases of advanced disease (Fisch IIIa/b). This allowed intra-operative repair of a cerebrospinal fluid leak created during surgery. In this series, there were two recurrences at 3 and 8 months post-operatively, both of which were excised using a second endoscopic approach.

\section{Endoscopic Surgery Alone}

There are increasing numbers of endoscopic surgery alone now appearing in the literature [9, 17-21]. However, few authors have large experience with very advanced disease and the consensus is that the endoscopic approach has limitations and is not suitable for tumours with extensive intra-cranial extension, extension lateral to the cavernous sinus, or posterior to the pterygoid plates $[9,17,19]$.

Mair et al. [20] report that the technique of using sublabial and buccolabial incisions permits lateral access to tumours using the endoscope. They also advocate the use of image guidance and the Nd YAG laser to photocoagulate the tumour prior to debulking with a microdebrider. In this series of 5 patients, the disease ranged from Fisch IIa to IIIa.

Mann et al. [9] comment on the use of the endoscope for Fisch III tumours, but it is not clear how many tumours of this size they have treated. Onerci et al. [19] discuss their experience of 12 cases, 4 of which were classed as Radowski's IIIa. In half of these cases, the tumour was not removed, as it was located around the cavernous sinus. These cases with residual tumour have not progressed in the 2 years of observed follow-up.

Three other series report the experience with the use of the endoscope alone. In the series of Wormald and Van Hasselt [18], none of 7 patients had a disease greater than Radowski's IIc; however, Nicolai et al. [17] had 4 of 15 patients with Fisch stage III disease. Nicolai comments on the importance of drilling out the vidian canal in preventing possible recurrence. This point is strongly emphasized by Howard et al. $[6,13]$ who claim to have dra- 
matically reduced their recurrence rate by exploring the basisphenoid to remove all tumours which had invaded the cancellous bone at the skull base. Roger et al. [21] report a series of 20 cases, 9 of whom were Radowski's types IIIa. All were treated endoscopically with complete excision achieved in $8 / 9$ cases (88\%). They recommend that embarking on this procedure, patients must be warned of the possibility of resorting to an open procedure. They report that the use of 2 experienced surgeons allows for the use of three hands in the operating field, which, in their opinion, considerably improves visualization and reduces the surgical time.

\section{Radiotherapy}

Two recent large series have been published using conventional radiotherapy $[22,23]$. Both series report local tumour control rates of $85 \%$.

Reddy et al. [22] treated 15 patients, 10 of which had Chandler stage IV disease. Nine patients had undergone previous surgery, and 6 had been treated by radiotherapy alone. The patients received between 30 and $35 \mathrm{~Gy}$ and were followed up by CT or MRI scanning. They were observed on imaging for tumour regression with/without stabilization of the persistent radiological abnormalities. Two patients required further treatment and both were salvaged by surgery. One patient developed a basal cell carcinoma of the skin within the radiation field 14 years after radiotherapy, 3 developed cataracts 5-10 years after radiotherapy, and 1 had a delayed, transient central nervous system syndrome.

The central nervous system syndrome [24] is an example of the clinical result from radiation damage to the brain. It may be acute, occurring within 5-6 h, or delayed - early, 2-4 months with encephalopathy, or late, months or years after radiation therapy, with reports of dementia, or possibly radiation-induced tumours.

Lee at al. [23] treated 27 cases with 3,000-5,500 cGy, of whom 23 (85\%) had Radowski's stage IIIb disease. They felt that in such advanced disease, the likelihood of fatal complications developing from the radiotherapy is less than the morbidity and mortality associated with surgery. They note that abnormal findings on post-treatment MRI are not unusual and do not necessarily indicate residual or recurrent disease. In 1 case with such findings, they performed fine-needle aspiration cytology, showing chronic inflammation only. They suggest that further treatment may not be indicated unless there are new radiological findings or recurrence of the pre-treatment symptoms. Four patients required further treatment, 2 by surgery and 2 by radiotherapy. One of the surgical cases required further radiotherapy to control the disease. Four patients suffered side effects including growth retardation, panhypopituitarism, temporal lobe necrosis, cataracts, and radiation keratopathy. The authors comment on the need to monitor endocrine function after treatment and the possible avoidance of such post-treatment sequelae with the development of more advanced techniques, such as CT conformal planning and intensitymodulated radiotherapy.

Intensity-modulated radiotherapy has been used by Kuppersmith et al. [25] to deliver 3,400-4,500 cGy to 3 patients with extensive disease involving the cavernous sinus and skull base. All tumours had been reduced in size on follow-up imaging and the only long-term side effects reported were epistaxis and rhinitis. Dare et al. [26] have used the gamma-knife (20-Gy dose) to treat 2 cases (Chandler stage III and IV) following incomplete surgical excision. The residual tumour remained unchanged in size $\left(4.7\right.$ and $3.0 \mathrm{~cm}^{3}$ ) at 3-year post-treatment follow-up. The authors cite the advantage of the gamma-knife as only 1 day of treatment, opposed to a course of radiotherapy lasting several weeks.

\section{Recurrence: Detection, Avoidance and Treatment}

It is clear that some authors are comfortable with leaving residual tumours in order to minimize morbidity [ 12 , $14,15]$, while others have been confident to proceed to total tumour removal $[10,16,27]$. Therefore, it is difficult to discuss tumour recurrence rates if part of the tumours are left (persistence of tumour at the time of surgery) without any further treatment. Some reports advocate routine radiotherapy for residual disease [26]; however, many others monitor the disease radiologically for several years, noting any stability or tumour progression [14, 19, 20], and indeed, complete regression of residual disease has been noted in 6 cases on serial CT scanning [13, 15]. The same philosophy holds true for managing patients after radiotherapy, as residual radiological findings do not necessarily indicate residual tumour, as noted earlier [23]. It has been reported [22] that regression of a tumour, residual or otherwise, may take as long as 3 years, and that tumours that persist in size after 2 years are likely to be still active. The primary reason for tumour recurrence is strongly believed to be the result of incomplete resection of the original tumour [28]. There is also a debate as to 
whether the cavernous sinus dura is invaded or just deformed by JNA tumour pressure; this is the area where the tumour is most frequently left by surgeons [29]. Such tumours require surgical experience, expertise, and the use of microscopic dissection.

Herman et al. [15] analysed recurrences in 44 cases treated surgically using a transfacial approach - lateral rhinotomy [25], midfacial degloving [16], or Le Fort I maxillary down-fracture osteotomy [1] - and a purely endoscopic approach in 2 cases. A neurosurgical approach was combined with the transfacial approach in 6 cases, and endoscopy was combined with the transfacial approach in a further 6 cases. Serial CT scanning was used to follow these patients and they reported that pre-operative tumour stage was a predictor of recurrence (a recurrence rate of $39.5 \%$ for Radowski's stage III compared with 7\% for stages II and I). In particular, recurrence was associated with extension into the following sites: infratemporal fossa, sphenoid, foramen lacerum, medial cavernous sinus, and anterior cranial fossa.

Mello-Filho et al. [27] report a series of 19 patients using the Le Fort I approach. The extent of each tumour is described, but no classification system is applied; however, $21 \%$ of the cases had intra-cranial extension. Over the follow-up period of $1-19$ years (mean 9.7 years) there were no stated recurrences. However, El-Banhawy et al. [16], using an endoscopically assisted midfacial degloving approach, noted 2 recurrences from 15 cases of Fisch IIIa/b, both of which were dealt with by an endoscopic resection. In endoscopic series, Mair et al. [20] and Nicolai et al. [17] have noted cases of recurrence; however, their experience with advanced disease is limited by their own admission, as this may be a contra-indication for a purely endoscopic technique.

Both recent radiotherapy series report tumour control in $85 \%[22,23]$. In both series, the recurrent tumour was salvaged with either further surgery, further radiotherapy, or in 1 case, both.

\section{To Embolize or Not?}

The vast majority of series reviewed employ pre-operative tumour embolization $24-48 \mathrm{~h}$ prior to surgery. The majority of authors feel that this significantly reduces intra-operative blood loss resulting in better surgical visualization. However, Lloyd et al. [6, 30] argue against this, stating that embolization can lead to poor tumour removal if there is deep invasion of the sphenoid; indeed, they state that this finding on pre-operative imaging is a contra-indication to embolization. Mann et al. [9] also raised concerns about increased recurrence following embolization.

Some authors quote recurrence rates leaving out cases with residual disease, if applicable. Not all authors state their follow-up protocol for monitoring their post-operative results, making direct comparisons between different series of treated patients difficult. Most would advocate serial imaging with endoscopic examination. Lloyd et al. [30] suggest the use of serial substitution MRIs to be the best form of radiological surveillance. If recurrences occur, they usually occur quickly, within 12-24 months [6, $8,16]$.

\section{Follow-Up Imaging after Treatment}

A suggested imaging protocol [31] for the follow-up of patients treated for JNA has been suggested. Most authors, when reporting their patient series, avoid commenting on the methods and intervals used in their posttreatment follow-up.

1 For patients who are clinically asymptomatic, if endoscopy is negative and no residual mass is visualized on radiographic examination 3-4 months following surgery, a clinical survey alone is recommended.

2 The clinical context is consistent with recurrent tumour if a mass is visualized on endoscopy, and CT or MRI suggest the diagnosis of recurrence/residual tumour, delineating its extensions. In this case, a second treatment should be performed - surgery or radiotherapy.

3 If the patient is asymptomatic, endoscopy is negative, and an enhanced mass outside the nasopharyngeal cavity is seen on the first radiological examination done 3-4 months after treatment, a new radiological examination is performed 3-6 months later:

a) If the mass is smaller and/or less enhanced, a third examination should be performed 6 months later. In case of absent evolution on the last examination, the follow-up will be clinical only.

b) If the mass is stable, a radiological follow-up every 6 months should be mandatory.

c) If the mass is increasing in size, a second surgery or radiotherapy is indicated. 


\section{Conclusions}

No single author or institution had a large series of advanced JNA. Most authors are comfortable with the concept of leaving the tumour behind in 'dangerous areas' for serial radiological monitoring, with the caveat of employing radiotherapy if there is evidence of disease progression. Although in most cases, no residual tumour progression has been observed.

Endoscopic techniques have greatly influenced the management of smaller tumours. To date, their role in treating larger tumours may be as an adjunct to standard open techniques. However, this may change as surgeons' experiences increase. Most authors comment that using the endoscope to examine the surgical cavity during tumour removal allows for more complete excision and improves access to key sites, such as the basisphenoid.

Radiotherapy achieves good control rates; however, the risk of side effects, including endocrine hypofunction and secondary malignancies, needs to be considered and explained to the patient. It is a likely possibility that this side effect may become less of a risk in the era of conformal and intensity-modulated radiotherapy. The role for the gamma-knife is unknown at present.

\section{References}

1 Andrews JC, Fisch U, Valavanis A, et al: The surgical management of extensive nasopharyngeal angiofibromas with the infratemporal fossa approach. Laryngoscope 1989;99:429-437.

$\checkmark 2$ Chandler JR, Goulding R, Moskowitz L, et al: Nasopharyngeal angiofibromas: staging and management. Ann Otol Rhinol Laryngol 1984; 93:322-329.

>3 Radowski D, McGill T, Healy GB, et al: Angiofibroma: changes in staging and treatment. Arch Otolaryngol Head Neck Surg 1996;122: 122-129.

4 Sessions RB, Bryan RN, Naclerio RM, et al: Radiographic staging of juvenile angiofibroma. Head Neck Surg 1981;3:279-283.

$\checkmark 5$ Sennes LU, Butugan O, Sanchez TG, et al: Juvenile nasopharyngeal angiofibroma: the routes of invasion. Rhinology 2003;41:235240

66 Lloyd G, Howard D, Phelps P, et al: Juvenile angiofibroma: the lessons of 20 years of modern imaging. J Laryngol Otol 1999;113:127134

7 Schick B, Urbschat S: New aspects of pathogenesis of juvenile angiofibroma. Hosp Med 2004;65:269-273

-8 Yadav SPS, Singh I, Chanda R, Sachdeva OP: Nasopharyngeal angiofibroma. J Otolaryngol 2002;31:346-350

-9 Mann WJ, Jecker P, Amedee RG: Juvenile angiofibromas: changing surgical concept over the last 20 years. Laryngoscope 2004; 114:291293.

-10 Bales C, Kotapka M, Loevner LA, et al: Craniofacial resection of advanced juvenile nasopharyngeal angiofibroma. Arch Otolaryngol Head Neck Surg 2002;128:1071-1078.

- 11 Fagan JJ, Snyderman CH, Carrau RL: Nasopharyngeal angiofibroma: selecting a surgical approach. Head Neck 1997;19:391-399.
12 Danesi G, Panizza B, Mazzoni A, et al: Anterior approaches in juvenile nasopharyngeal angiofibromas with intracranial extension. Otolaryngol Head Neck Surg 2000;122:227-283.

13 Howard DJ, Lloyd G, Lund V: Recurrence and its avoidance in juvenile angiofibroma. Laryngoscope 2001;111:1509-1511.

14 Zhang M, Garvis W, Linder T, et al: Update on the infratemporal fossa approaches to nasopharyngeal angiofibroma. Laryngoscope 1998; 108:1717-1723.

-15 Herman P, Lot G, Chapot R, et al: Long-term follow-up of juvenile nasopharyngeal angiofibromas: analysis of recurrence. Laryngoscope 1999; 109:140-147.

16 El-Banhawy OA, El-Dien AE, Amer T: Endoscopic-assisted midfacial degloving approach for type III juvenile angiofibroma. Int J Pediatr Otolaryngol 2004;68:21-28.

17 Nicolai P, Berlucchi M, Tomenzoli D, et al: Endoscopic surgery for juvenile angiofibroma: when and how. Laryngoscope 2003;113:775782 .

18 Wormald PJ, Van Hasselt A: Endoscopic removal of juvenile angiofibromas. Otolaryngol Head Neck Surg 2003;129:684-691.

19 Onerci TM, Yucel OT, Ogretmenoglu O: Endoscopic surgery in treatment of juvenile nasopharyngeal angiofibroma. Int J Pediatr Otolaryngol 2003;67:1219-1225.

20 Mair EA, Battiata A, Casler JD: Endoscopic laser-assisted excision of juvenile nasopharyngeal angiofibromas. Arch Otolaryngol Head Neck Surg 2003;129:454-459.

21 Roger G, Ba-Huy PT, Froehlich P, et al: Exclusive endoscopic removal of juvenile nasopharyngeal angiofibroma. Arch Otolaryngol Head Neck Surg 2002;128:928-935
22 Reddy KA, Mendenhall WM, Amdur RJ, et al: Long-term results of radiation therapy for juvenile nasopharyngeal angiofibroma. Am J Otolaryngol 2001;22:172-175.

23 Lee JT, Chen P, Safa A, et al: The role of radiation in the treatment of advanced juvenile angiofibroma. Laryngoscope 2002;112:12131220

24 Beers MH, Berkow R: Merck Manual of Diagnosis and Therapy. Merck website: http:// www.merck.com/mrkshared/CVMHigh Light?file $=/ \mathrm{mrkshared} / \mathrm{mmanual} / \mathrm{section} 14 /$ chapter177/177f.jsp.

25 Kuppersmith RB, The BS, Donovan DT, et al: The use of intensity modulated radiotherapy for the treatment of extensive and recurrent juvenile angiofibroma. Int J Pediatr Otolaryngol 2000;52:261-268.

$>26$ Dare AO, Gibbons KJ, Proulx GM, et al: Resection followed by radiosurgery for advanced juvenile nasopharyngeal angiofibroma: report of two cases. Neurosurg 2003;52:1207-1211.

$>27$ Mello-Filho FV, De Freitas LCC, Santos ACD, et al: Resection of juvenile angiofibroma using the Le Fort 1 approach. Am J Otolaryngol 2004;25:157-161.

-28 Liu L, Wang R, Heung D, et al: Analysis of intraoperative bleeding and recurrence of juvenile nasopharyngeal angiofibroma. Clin Otolaryngol 2002;27:536-540.

29 Donald PJ, Enepikedes D, Boggan J: Giant juvenile nasopharyngeal angiofibroma: management by skull-base surgery. Arch Otolaryngol Head Neck Surg 2004; 130:882-886.

30 Lloyd G, Howard D, Lund VJ, et al: Imaging for juvenile angiofibroma. J Laryngol Otol 2000; 114:727-730.

-31 Chagnaud Ch, Petit PH, Bartoli JM, et al: Postoperative follow-up of juvenile nasopharyngeal angiofibromas: assessment by CT scan and MR imaging. Eur Radiol 1998;8:756-764. 